\title{
Pragmatische Organisationsgestaltung im Kontext von Branche und Land: Manager in Polen, Ostdeutschland und Tschechien im betrieblichen Transformationsproze ${ }^{*}$
}

\author{
Ralph-Elmar Lungwitz, Evelyn Preusche ${ }^{* *}$
}

Eine differenzierte Betrachtung der Rolle von Managern im betrieblichen Transformationsprozeß erfordert nicht nur die Berücksichtigung länderspezifischer Entwicklungspfade, sondern auch branchenspezifisch differenzierter struktureller Handlungsbedingungen und Reformbedarfe. Im vorliegenden Beitrag werden auf der Basis von Fallstudien typische Entwicklungen der Unternehmens- und Arbeitsorganisation in der Textil/Bekleidungsindustrie, dem Maschinenbau und der Kraftfahrzeugzulieferindustrie in Polen, Ostdeutschland und Tschechien aufgezeigt und es wird nach der Rolle der Manager bei der Genese dieser Strukturen gefragt. Das für die Mehrzahl der Manager charakteristische pragmatische Verhältnis zu der Gestaltung betrieblicher Organisation führte in den Branchen aufgrund spezifischer Bedingungen zu unterschiedlichen Ergebnissen.

A complex examination of the role of managers within processes of enterprise transformation does not only call for the consideration of national paths of development but also of specific structural conditions of acting and of reform requirements on the level of branches. In the submitted article based on case studies typical patterns of firm and work organisation in the textile/clothing industries, in mechanical engineering and in the component manufacturing for motor vehicles in East Germany, Poland and the Czech Republic are discussed and it is being asked for the role of managers in the emergence of these structures. The pragmatic attitude towards organisational change characteristic for the majority of managers led to different outcomes according to specific conditions in the included branches.

\footnotetext{
* manuscript received: 27.9.1999, accepted: 18.2.2000;

** Ralph-Elmar Lungwitz, 1951, WISOC e.V., Forschungsfelder: Industriesoziologie, Management, Organisation; Evelyn Preusche, 1957, WISOC e.V., Forschungsfelder: Industriesoziologie, Management, Organisation;
} 


\section{Differenzierte Wege bei der Transformation organisatorischer Handlungsfähigkeit}

Der Systemwechsel in Mittel- und Osteuropa schien zeitlich mit dem Beginn eines Paradigmenwechsels innerhalb des westlichen Produktionsmodells zusammenzufallen. Das Leitbild der tayloristischen Massenproduktion sollte durch flexiblere organisatorische Lösungen ersetzt werden, die nicht zuletzt für die Beschäftigten auf der Shop-Floor-Ebene größere Handlungsspielräume und eine stärkere Mitverantwortung vorsahen. Während sich diese Verheißung zunächst mit dem Programm von „Lean-Produktion“ verband, brachte die Beraterindustrie später immer neue Konzepte ${ }^{1}$ auf den Markt, deren Anspruch darauf, die einzig angemessene Antwort auf Globalisierung und gesellschaftliche Modernisierung darzustellen, mit immer kürzeren „Halbwertzeiten“ verbunden war. Aus Gründen, die hier nicht im Einzelnen dargestellt werden können, hat in den letzten Jahren der Glaube an ein neues universelles Leitbild „moderner“ Arbeits- und Organisationsgestaltung bei Theoretikern und Praktikern deutlich nachgelassen. Inwieweit man darin einen Verlust für die praktizierenden Manager in den Transformationsländern sieht, sei dahingestellt, auf alle Fälle entfällt für die Wirtschafts- und Sozialforscher die Möglichkeit des Gebrauchs komplexitätsreduzierender Begriffe wie „strukturkonservative“ oder „strukturinnovative“ Organisation. Der Verschleiß eines normativen Leitbildes "moderner" Arbeits- und Organisationsgestaltung in der Marktwirtschaft zwingt die Manager zu einer eigenständigen Suche nach Lösungen, die den konkreten Bedingungen des jeweiligen Unternehmens entsprechen, und sie bedeutet für die Wissenschaftler die Herausforderung, diesen Prozeß zu rekonstruieren, ohne sich in der Beschreibung individueller Einzelfälle zu erschöpfen. Wenn kein normatives Leitbild mehr existiert, dann können auch die Kriterien dieses Leitbildes nicht mehr als Maßstab benutzt werden, um den gegebenen Zustand eines Unternehmens zu beurteilen, sondern dann ist zunächst nach der Funktionalität einer gegebenen Arbeits- und Organisationsgestaltung angesichts konkreter Bedingungen zu fragen. ${ }^{2}$

Eine konkrete Analyse des betrieblichen Transformationsprozesses schließt auch eine differenzierte Betrachtung der Rolle des Managements ein. Strukturelle Handlungsbedingungen, Situationsdefinitionen sowie Verlauf und Ergebnisse organisationsgenerierenden Handelns der Manager bilden betriebsindividuelle Konstellationen, die aber auch in branchen- und länderspezifische Zusammenhänge eingebettet sind. Zwar ist es auch in Bezug auf die betriebliche Transformation prinzipiell richtig, daß die Manager mehr sind, ,als

${ }^{1}$ Wir denken dabei u.a. an Konzepte wie TQM, Business Reengineering, Gruppenarbeit, Bildung von Cost- und Profitcentern oder Empowerment.

${ }^{2}$ Dies schließt natürlich eine Bewertung der Situation etwa aus der Sicht der abhängig Beschäftigten nicht aus. 
Vollzugsorgane einer durch die jeweilige Umwelt vorgegebenen Gestaltungslogik“ (Schienstock 1993:13), jedoch resultieren aus der betrieblichen Umwelt mitunter sehr eindeutige Prämissen für die organisatorische Gestaltung, deren Mißachtung durch die Manager einer „falschen“ Situationsdefinition (Esser 1996) mit verhängnisvollen Folgen für das Unternehmen oder auch für die betreffenden Manager selbst gleichkäme, so daß man in diesen Fällen in der Tat von einer durch die Umwelt ,vorgegebenen Gestaltungslogik“ sprechen muß. Auch ist $\mathrm{zu}$ bedenken, daß organisationsbezogene Aspekte nur einen Teilbereich einer komplexeren Situationsdefinition der Manager ausmachen, da organisationsgestaltende Tätigkeit nur einen Teil managerieller Praxis darstellt. Organisatorische Sachverhalte müssen im Rahmen ihrer Gesamtverantwortung für die Manager eine subjektive Bedeutsamkeit als aktuelles oder potentielles Problem erhalten, um eine intentionale Gestaltung überhaupt erst in Gang zu setzen, anderenfalls bleibt die Organisation Teil der ,taken-for-granted-world“, der schon aus Gründen einer die Manager entlastenden Komplexitätsreduktion keine relevante Bedeutung beigemessen wird.

Bisherige Untersuchungen zu institutionellen und wirtschaftlichen Aspekten des Transformationsprozesses heben insbesondere die notwendigen Veränderungen im Denken und Handeln der Manager sowie im Aufbau und der Funktionsweise von Unternehmen hervor, die sich aus den Unterschieden zwischen Plan- und Marktwirtschaft ergeben und verweisen auf die problematischen Folgen weiterbestehender kultureller Prägungen und institutioneller Hinterlassenschaften des alten Systems. Eine Betrachtungsweise, die sich auf den qualitativen Umbruch des Systemwechsels konzentriert und vor allem die Last des Erbes des Ancien régime in den Mittelpunkt stellt, war in den ersten Jahren des Transformationsprozesses sicherlich gerechtfertigt. Heute muß man allerdings der Tatsache Rechnung tragen, daß Manager und andere Akteure im wesentlichen unter Bedingungen handeln, die im Verlauf einer nunmehr zehnjährigen Geschichte innerhalb des neuen Systems entstanden sind und sich verändert haben. Die Erlangung und Beibehaltung organisationaler Handlungsfähigkeit von Unternehmen ist deshalb zumindest in den fortgeschritteneren Transformationsländern, in denen sich funktionsfähige Marktwirtschaften herausgebildet haben ${ }^{3}$, nicht mehr in erster Linie als Überwindung des problematischen Erbes der Planwirtschaft zu verstehen, sondern muß mit Bezug auf die aktuellen Bedingungen und Herausforderungen thematisiert werden. Dies schließt die Bestimmung des differenzierten Reformbedarfes ein, der bei der Erlangung oder Bewahrung organisationaler Handlungsfähigkeit von Unternehmen im Transformationsprozeß durch das

$3 \mathrm{Zu}$ dieser Einschätzung gelangt die EU-Kommission in ihrer Stellungnahme zu den Beitrittsanträgen Polens und der Tschechischen Republik. 
Management und andere betriebliche und außerbetriebliche Akteure zu bewältigen war. Betriebsgröße, Grad der Strategiefähigkeit, die Stellung als Finalist oder Zulieferer, Eigentumsverhältnisse einschließlich der Rolle westlichen Kapitals, externe Flexibilitätsanforderungen, die Arbeitsmarktsituation und eine Hoch- bzw. Niedriglohnoption stellen wesentliche differenzierende Faktoren in Bezug auf die organisatorische Handlungsfähigkeit unter marktwirtschaftlichen Bedingungen und die Wege ihrer Transformation dar. Neben der Berücksichtigung länderspezifischer Besonderheiten bedarf es insbesondere einer branchenbezogenen Analyse, da die oben genannten Merkmale sowohl in länder- als auch in branchenspezifischen Konstellationen auftreten. Eine differenzierte Betrachtung des zu bewältigenden Reformbedarfes auf der Ebene konkreter Unternehmen schließt die Aufgabe ein, deutlich zu machen, welche Komponenten der Organisation durch das Management intentional geändert werden mußten, um die organisationale Handlungsfähigkeit des Unternehmens unter den konkreten Bedingungen herzustellen, welche Komponenten im wesentlichen beibehalten werden konnten und in welcher Hinsicht das Management auf spontane Anpassungsprozesse im Verhalten betrieblicher Akteure und in der betrieblichen Sozialverfassung setzen konnte, die sich aus geänderten externen Rahmenbedingungen ergaben.

\section{Die Untersuchung}

Die Rolle von Managern in Industriebetrieben Ostdeutschlands, Polens und der Tschechischen Republik bei der Transformation der Unternehmens- und Arbeitsorganisation war das Thema eines Forschungsprojektes am Institut für Wirtschafts- und Sozialforschung Chemnitz ${ }^{4}$, das von der Volkswagen Stiftung gefördert wurde. Das Ziel der Untersuchung bestand darin, herauszufinden, mit welcher Gestaltung der Unternehmens- und Arbeitsorganisation ostdeutsche, polnische und tschechische Unternehmen nach Abschluß auf gegenwärtige Anforderungen reagieren, und welche Rolle vor allem die Manager bei der Herausbildung der aktuellen Organisationsstrukturen spielten. Wir interessierten uns insbesondere dafür, welche Aspekte der Organisation von den Managern im Rahmen einer komplexeren Situationsdefinition und einer darauf basierenden

${ }^{4}$ Dem Projektteam gehörten außer den Autoren dieses Beitrages noch an: Ivana Hollerova, Karlsuniversität Prag, Jan Vlacil, Wirtschaftsuniversität Prag, Maria Gagacka und Anatol Peretiatkowicz von der Privaten Universität für Umweltschutz in Radom. 
unternehmerischen Strategie als problematisch und damit veränderungswürdig betrachtet wurden und zu welchen Resultaten dies führte. ${ }^{5}$

Bei der Auswahl der Untersuchungsbetriebe konzentrierten wir uns von vornherein auf drei Branchen, um diese in der Transformationsforschung bisher eher vernachlässigte Dimension angemessen zu berücksichtigen ${ }^{6}$, und zwar die Kraftfahrzeugzulieferindustrie, den Maschinenbau und die Textil/Bekleidungsindustrie. Dabei handelte es sich nicht nur um Branchen, die die Industriestruktur der einbezogenen Länder traditionell prägten und dies auch noch heute tun, sondern auch um Branchen, die deutliche Differenzierungen hinsichtlich der Problemstellung des Projektes erwarten ließen. Bereits an dieser Stelle sei vorweggenommen, daß sich unsere These von einer Branchenspezifik organisationsgenerierenden Handelns von Managern und damit auch ihrer Resultate voll bestätigte.

Pro Branche und Land nahmen wir zwei Betriebe in unser Sample auf. Einen allgemeinen Überblick über die Untersuchungsbetriebe geben die folgenden Tabellen.

Unsere Untersuchungsbetriebe aus dieser Branche stellen durchweg relativ einfache Komponenten her, mit denen sie die Finalisten direkt, zum Teil Just-InTime, beliefern. Durch das Engagement der Eigentümer gelang es fast allen unseren Untersuchungsbetrieben, mit Ausnahme der polnischen Genossenschaft KFZ/PL-1, sich einen Platz in den sich neu formierenden nationalen Zuliefernetzwerken zu sichern und darüber hinaus auch in einigen Fällen ihre Erzeugnisse zu exportieren. Es wäre falsch, sie als reine Fertigungsstätten zu

${ }^{5}$ Das Projekt basierte auf Fallstudien in 18 Betrieben (je 6 pro Land), die ihre Ursprünge in der Planwirtschaft hatten. In diesen Untersuchungsbetrieben führten wir zwischen Frühjahr 1998 und Frühjahr 1999 insgesamt 156 problemzentrierte qualitative Interviews mit den Geschäftsführern, mit den Leitern betrieblicher Funktionalbereiche, mit Meistern, Belegschaftsvertretern und Vertretern privater Eigentümer durch. Diese betrieblichen Interviews wurden ergänzt durch Expertengespräche mit den Vertretern von Gewerkschaften, Kammern und Wirtschaftsverbänden. Dies gelang allerdings in Ostdeutschland besser als in Tschechien und Polen, was sicherlich auch mit der relativ schwachen Etablierung dieser intermediären Institutionen in den MOE-Ländern im Vergleich zur Situation in Deutschland zusammenhängt.

${ }^{6}$ Es ist verständlich, daß bei einer Perspektive auf den Transformationsprozeß, die vor allem die qualitativen Differenzen zwischen Plan- und Marktwirtschaft in den Mittelpunkt stellt, Branchenunterschiede nur eine untergeordnete Rolle spielen. Daß die Branchenspezifik hinsichtlich der Arbeits- und Organisationsgestaltung von Belang ist, machten ja auch die Diskussionen über die Universalität des Taylorismus (z.B. Labour Process Debate) oder über die Generalisierbarkeit von Lean-Production deutlich. Einzelne Untersuchungen befaßten sich auch mit der Transformation von Branchen in Ostdeutschland (z.B. Mickler et al. 1996, Lungwitz 1996) 
charakterisieren, allerdings ist ihre unternehmerische Autonomie eingeschränkt, was nicht ohne Folgen für die Gestaltung betrieblicher Funktionalbereiche wie Konstruktion, Einkauf, Marketing und Vertrieb bleibt, da diese Aufgaben zumindest teilweise durch die Mutterfirmen oder auch durch die wichtigsten Kunden selbst wahrgenommen werden.

Tabelle 1: Untersuchungsbetriebe der Kraftfahrzeugzulieferindustrie

\begin{tabular}{|l|c|l|l|}
\hline Bezeichnung & Beschäftigte & \multicolumn{1}{|c|}{ Erzeugnisse } & \multicolumn{1}{|c|}{ Eigentümer } \\
\hline OD/Kfz-1 & 600 & $\begin{array}{l}\text { mechanische } \\
\text { Baugruppen }\end{array}$ & Konzern \\
\hline OD/Kfz-2 & 193 & $\begin{array}{l}\text { einfache mech. } \\
\text { Baugruppen }\end{array}$ & $\begin{array}{l}\text { bisher: westdeutsches } \\
\text { Familienunternehmen; } \\
\text { Übernahme durch nord- } \\
\text { amerikanisches Unternehmen } \\
\text { geplant }\end{array}$ \\
\hline TR/Kfz-1 & 315 & $\begin{array}{l}\text { Plastikteile } \\
\text { Ferkzeug- u. } \\
\text { Formenbau }\end{array}$ & $\begin{array}{l}46 \% \text { tschech. Privatperson } \\
41 \% \text { ausländ. Unternehmen }\end{array}$ \\
\hline TR/Kfz-2 & 250 & $\begin{array}{l}\text { Sitze f. LKW, } \\
\text { Busse, Züge }\end{array}$ & $\begin{array}{l}\text { Handelsgesellschaft im Besitz } \\
\text { einer tschech. Privatperson }\end{array}$ \\
\hline PL/Kfz-1 & 115 & $\begin{array}{l}\text { Sitze f. LKW; } \\
\text { Planen, } \\
\text { Bordwände }\end{array}$ & Genossenschaft \\
\hline PL/Kfz-2 & 610 & $\begin{array}{l}\text { PKW-Sitze, } \\
\text { Spezialfahrzeuge }\end{array}$ & asiatischer PKW-Hersteller \\
\hline
\end{tabular}

Mit Ausnahme des existenzgefährdeten polnischen Unternehmens PL/Kfz-1 wurde die wirtschaftliche Situation der Untersuchungsbetriebe dieser Branche ebenso wie deren technische Ausstattung, die im Ergebnis von Modernisierungsinvestitionen der Eigentümer erfolgte, von den Managern als gut bis sehr gut eingeschätzt. Dabei handelt es sich mit Sicherheit um eine allgemeine Tendenz: Die sich entwickelnde Automobilindustrie in den MOELändern zieht ausländisches Kapital an und dieses ist auch bereit und in der Lage, die erforderlichen Investitionen vorzunehmen.

Die in unsere Untersuchung einbezogenen polnischen, tschechischen und ostdeutschen Maschinenbaufirmen weisen hinsichtlich ihrer Situation vor Beginn der Transformation eine große Ähnlichkeit auf. Es handelte sich bei ihnen um hierarchisch strukturierte und funktional ausdifferenzierte 
Großbetriebe oder Teile davon, die als Finalproduzenten eine bedeutende Stellung im Rahmen der nationalen Volkswirtschaften innehatten. Hauptabnehmer ihrer Erzeugnisse waren die Länder des ehemaligen RGW, insbesondere die Sowjetunion. Alle Firmen waren mit ihren Erzeugnissen bereits vor 1989 auch auf westlichen Märkten präsent. Der Zusammenbruch der Ostmärkte nach 1990, die Sättigung der Nachfrage auf den Binnenmärkten und der Wegfall vieler einheimischer Kunden führte in Verbindung mit der weltweiten Rezession im Maschinenbau Anfang der 90er Jahre zu gravierenden Marktverlusten und Auftragseinbrüchen und belastete die beginnende marktwirtschaftliche Restrukturierung nachhaltig.

Tabelle 2: Untersuchungsbetriebe des Maschinenbaus

\begin{tabular}{|l|c|l|l|}
\hline Bezeichnung & Beschäftigte & \multicolumn{1}{|c|}{ Erzeugnisse } & \multicolumn{1}{|c|}{ Eigentümer } \\
\hline OD/MB-1 & 210 & Textilmaschinen & $\begin{array}{l}\text { westdeutsches } \\
\text { Familienunternehmen }\end{array}$ \\
\hline OD/MB-2 & 105 & $\begin{array}{l}\text { Werkzeug- } \\
\text { maschinen }\end{array}$ & $\begin{array}{l}\text { Finanzgesellschaft, Verkauf an } \\
\text { eine ausländische } \\
\text { Firmengruppe geplant }\end{array}$ \\
\hline TR/MB-1 & 500 & $\begin{array}{l}\text { Werkzeug- } \\
\text { maschinen }\end{array}$ & $\begin{array}{l}\text { Mehrheitsanteile bei } \\
\text { Handelsgesellschaft }\end{array}$ \\
\hline TR/MB-2 & 670 & $\begin{array}{l}\text { Werkzeug- } \\
\text { maschinen }\end{array}$ & $\begin{array}{l}\text { MbO; 3 Manager halten 97 \% } \\
\text { der Anteile }\end{array}$ \\
\hline PL/MB-1 & 4420 & Baumaschinen & Holding im Staatsbesitz \\
\hline PL/MB-2 & 124 & $\begin{array}{l}\text { innerbetriebliche } \\
\text { Transport- } \\
\text { einrichtungen }\end{array}$ & $\begin{array}{l}80 \% \text { staatliches Unternehmen } \\
20 \% \text { Mitarbeitergesellschaft }\end{array}$ \\
\hline
\end{tabular}

Mitte der 90er Jahre war in den untersuchten Betrieben die Anpassung an die marktwirtschaftlich ausgerichteten Rahmenbedingungen weitgehend vollzogen. Während die tschechischen und ostdeutschen Maschinenbaubetriebe auch nach der Privatisierung über eigene Marketing- sowie Entwicklungs- und Konstruktionsabteilungen verfügten und damit strategiefähig blieben, wurden diese Bereiche von den Eigentümern der polnischen Untersuchungsbetriebe (in beiden Fällen sind dies Staatsbetriebe) als eigenständige Firmen ausgegründet. Die befragten polnischen Manager sehen in der ihnen fehlenden Möglichkeit, auf die Marktarbeit und die Konstruktion Einfluß nehmen zu können, und der strukturellen Abhängigkeit der formell unabhängigen Firmen von den Eigentümern eine entscheidende Ursache für die schlechte Situation der 
Firmen. $^{7}$ Hinzu kommt, daß der polnische Maschinenbau nicht jene Anziehungskraft für private Investoren hatte wie die Automobilindustrie. Damit fehlte den kommerzialisierten Betrieben dringend benötigtes Kapital, insbesondere für die Modernisierung der Technik. Die ostdeutschen und tschechischen Maschinenbaubetriebe konnten hingegen nach Mitte der 90er Jahre von der positiven Entwicklung der Branche insgesamt profitieren. Vor allem in Ostdeutschland verfügen die Maschinenbaufirmen dank umfangreicher Investitionen der Eigentümer über eine moderne technische Ausstattung. Die tschechischen Manager bewerten die technische Ausstattung ihrer Firmen als ausreichend oder gut, können sich allerdings auch nicht auf finanzkräftige Eigentümer stützen.

Tabelle 3: Untersuchungsbetriebe der Textil- und Bekleidungsindustrie

\begin{tabular}{|l|c|l|l|}
\hline Bezeichnung & Beschäftigte & \multicolumn{1}{|c|}{ Erzeugnisse } & \multicolumn{1}{|c|}{ Eigentümer } \\
\hline OD/TB-1 & 105 & $\begin{array}{l}\text { Unterwäsche } \\
\text { Sport- und } \\
\text { Freizeit- } \\
\text { bekleidung }\end{array}$ & $\begin{array}{l}\text { Privatperson aus } \\
\text { Westdeutschland }\end{array}$ \\
\hline OD/TB-2 & 120 & $\begin{array}{l}\text { Bett- u. Tisch- } \\
\text { wäsche, Damast }\end{array}$ & $\begin{array}{l}\text { 2 ostdeutsche Privatpersonen; } \\
\text { reprivatisiert }\end{array}$ \\
\hline TR/TB-1 & 420 & Unterwäsche & $\begin{array}{l}\text { MBO-privatisiert, 3 Manager } \\
\text { und ein Jurist sind Eigentümer }\end{array}$ \\
\hline TR/TB-2 & 1700 & Strickgarn & Investmentfonds \\
\hline PL/TB-1 & 324 & Oberbekleidung & Polnische Privatperson \\
\hline PL/TB-2 & 247 & $\begin{array}{l}\text { Arbeits- } \\
\text { bekleidung } \\
\text { Handschuhe } \\
\text { Handtücher }\end{array}$ & Polnische Privatperson \\
\hline
\end{tabular}

Mit Ausnahme des tschechischen Textilbetriebes TR/TB-2 wurde die wirtschaftliche Situation aller untersuchten Betriebe der Textil- und Bekleidungsbranche von den Eigentümern als gut oder sehr gut eingeschätzt. Die Schwierigkeiten, denen sich TR/TB-2 gegenüber sieht, sind durchaus typisch für Produzenten der tschechischen Textilbranche, die textile Materialien

7 Das Management beider polnischen Maschinenbaufirmen beurteilte während unserer Erhebungen 1998 die wirtschaftliche Lage als schlecht bzw. sehr schlecht, PL/MB-2 droht der Bankrott. 
(Stoffe, Garne) zur weiteren Verarbeitung herstellen, da der Binnenmarkt hier weitgehend gesättigt ist. Dank umfangreicher Investitionen der Eigentümer der polnischen und ostdeutschen Betriebe verfügen diese über eine moderne technische Ausstattung, in der eine unabdingbare Voraussetzung für die Gewährleistung der von den Kunden gestellten Qualitätsanforderungen gesehen wird. Die technische Ausstattung der tschechischen Firmen wird als ausreichend, in bestimmten Bereichen des Großbetriebes TR/TB-2 auch als sehr modern bewertet.

\section{Entwicklungstendenzen der Unternehmens- und Arbeitsorganisation und die Rolle der betrieblichen Manager}

\subsection{Kraftfahrzeugzulieferindustrie}

Die Kraftfahrzeugindustrie gehört $\mathrm{zu}$ den Branchen in den ehemals sozialistischen Ländern, die sich nach Überwindung der Transformationskrise und der globalen Rezession 1992/93 am schnellsten erholten, was zweifellos auf das Engagement global operierender Fahrzeughersteller und Zulieferfirmen zurückzuführen ist. Ostdeutschland, Polen und Tschechien profitierten sowohl von der Errichtung moderner Montagewerke als auch von der Entstehung regionaler Zuliefernetzwerke ${ }^{8}$. Darüber hinaus wurden auch Zulieferunternehmen in Polen und Tschechien verstärkt mit der Lohnfertigung von Komponenten für westliche Zulieferer beauftragt. Mit der Einbindung in die neu entstehenden Zuliefernetzwerke, meist verbunden mit der Privatisierung, war die Frage des Marktzuganges für die Firmen prinzipiell gelöst. Die Manager konnten sich jetzt darauf konzentrieren, die technischen, organisatorischen und qualifikatorischen Voraussetzungen dafür zu schaffen, die meist sehr eindeutig formulierten Anforderungen ihrer Kunden hinsichtlich Kosten, Zeit und Qualität zu erfüllen. Betrachtet man insbesondere die Transformation der Unternehmensund Arbeitsorganisation in den Untersuchungsbetrieben, so lassen sich idealtypisch zwei Konstellationen unterscheiden: Es gibt Fälle, in denen ein international agierendes Mutterunternehmen sein Tochterunternehmen konsequent nach den Grundsätzen der Lean-Programmatik umgestaltet (OD/KFZ-1, PL/KFZ-2), und es gibt Fälle, in denen das betriebliche Management die organisatorische Restrukturierung weitgehend autonom betreibt, weil ihm der institutionelle Eigentümer diese Autonomie einräumt

${ }^{8}$ Dies gilt allerdings für die PKW-Produktion in wesentlich stäkerem Maße, als für die Herstellung von LKW. Zwar errichteten westliche LKW-Hersteller auch Montagewerke, sie verzichteten aber weitgehend auf die Einbeziehung regionaler Zulieferer und griffen statt dessen auf die Zulieferer aus ihren Herkunftsländern zurück. 
(OD/KFZ-2) oder weil der Geschäftsführer selbst der Eigentümer ist (die beiden tschechischen Betriebe).

\section{a) „coercive“ Restrukturierung durch den Mutterkonzern}

Auffallend ist, daß sich in den Untersuchungsbetrieben, die sich im Besitz global agierender Konzerne befinden, die Eigentümer auf durchaus „coercive“ Weise konkrete Konzepte der Organisationsgestaltung und des Einsatzes der Beschäftigten vorgaben und durchsetzten (Costcenterstruktur und Gruppenarbeit im Fall von OD/KFZ-1, betriebliches Vorschlagswesen, Innovations- und Problemlösungsgruppen in beiden Untersuchungsbetrieben). Sie stellten auch in beiden Fällen Führungskräfte aus ihren eigenen Reihen bereit, die vor Ort diese Konzepte umsetzten. Das örtliche Management wurde in die Erarbeitung der Details einbezogen. In beiden Fällen versuchen die Eigentümer, ihre organisatorischen Modernisierungskonzepte mit einer Art kultureller Hegemonie in den Unternehmen zu verankern. Sie legen großen Wert auf Visualisierungen, Losungen, Ordnung, Sauberkeit, Ästhetik. Hinsichtlich der betrieblichen Reorganisation wurde den Managern eine bestimmte Problemdefinition und darauf zugeschnittene Lösungen normativ vorgegeben. Sie verhielten sich gegenüber diesen Erwartungen der Eigentümer loyal, allerdings ließen die durchgeführten Interviews durchaus gewisse Differenzierungen hinsichtlich ihrer Einstellung $\mathrm{zu}$ diesen vorgegebenen Konzepten erkennen, die von euphorischer Zustimmung („Ich fühle mich total empowered“, Leiter Vertrieb OD/KFZ-1) bis hin zu einer gewissen Unzufriedenheit reichten. Es wurde deutlich, daß ein Teil der Vorbehalte denen gleicht, die auch aus westlichen Unternehmen bekannt sind: Die Dezentralisierung von Leitungskompetenzen stößt auf Akzeptanzprobleme bei den Führungskräften, die Kompetenzen abgeben sollen, aber auch diejenigen, die diese Kompetenzen im Ergebnis der Reform mit übernehmen sollen, zeigen angesichts wachsender Aufgaben und steigender Verantwortung häufig genug nur wenig Begeisterung. Es gibt freilich auch transformationstypische Widerstände gegen derartige Konzepte, die aus der formellen Ähnlichkeit einiger neu eingeführter Praktiken mit Erfahrungen aus der Planwirtschaft resultieren. ${ }^{9}$ Andererseits scheinen sich vor allem diejenigen betrieblichen Führungskräfte mit den organisatorischen Modernisierungskonzepten der Eigentümer zu identifizieren, die damit die Erfahrung eines beruflichen Aufstiegs und größerer persönlicher Entfaltungsmöglichkeiten verbinden.

${ }^{9}$ So wird der Kontinuierliche Verbesserungsprozeß häufig mit dem Neurerwesen assoziiert und die Visualisierung von Ergebnissen ruft Erinnerungen an den sozialistischen Wettbewerb wach. 
Natürlich lassen sich zwischen diesen beiden Fällen auch Unterschiede ausmachen, die auf länderspezifische Besonderheiten zwischen Polen und Ostdeutschland, wohl aber auch auf unterschiedliche Konzepte der Mutterkonzerne verweisen. Im Fall des ostdeutschen Betriebes verfolgte der aus dem angloamerikanischen Raum stammende Eigentümer (Hauptgesellschafter ist ein Pensionsfonds) seit der Übernahme des Werkes eine Politik der rigorosen Kostensenkung, die sich u.a. in mehreren Wellen des Personalabbaus niederschlug. Das der organisatorischen Modernisierung nach der LeanPhilosophie innewohnende Rationalisierungspotential wurde voll genutzt. ${ }^{10}$ Der asiatische Eigentümer des polnischen Zulieferbetriebes verfolgt dagegen das langfristige Ziel, seinen polnischen Tochterbetrieb $\mathrm{zu}$ einem führenden Hersteller von PKW-Sitzen für den europäischen Markt zu entwickeln. Er verzichtete auf Entlassungen, was ihm sicherlich durch die relativ geringen Löhne erleichtert wurde, obwohl die Produktionskapazitäten bei weitem nicht ausgelastet sind und er die betriebliche Verwaltung als $\mathrm{zu}$ groß und wenig effizient einschätzt. Die überkommene Organisationsstruktur wurde im wesentlichen beibehalten, lediglich Logistik und Lagerwirtschaft wurden umgestaltet, um den Anforderungen des JIT-Betriebes gerecht zu werden. Höchste Priorität wird durch den Eigentümer der weiteren Qualifikation der Mitarbeiter und der Festigung ihrer Verbundenheit mit der Firma beigemessen. Es werden Qualitätszirkel und regelmäßige Gesprächsgruppen zu betrieblichen Problemen durchgeführt, deren Hauptziel erklärtermaßen nicht in den wirtschaftlichen Effekten, sondern in einer besseren sozialen Integration der Mitarbeiter in die Firma besteht. Im Betrieb OD/KFZ-1 dagegen wird das Vorschlagswesen von den Leitern der Costcenter auf der Grundlage von Zielvorgaben straff und ergebnisorientiert geführt. Zahl und Ergebnisse der Vorschläge fließen in das interne Benchmarking und in die Leistungsbewertung der Costcenterleiter durch die Geschäftsführung ein.

Festzuhalten bleibt jedoch, daß sich sowohl in dem polnischen, als auch in dem ostdeutschen Untersuchungsbetrieb dieses Typs an der Arbeitsorganisation in der unmittelbaren Produktion relativ wenig geändert hat. Zwar wurde in dem ostdeutschen Betrieb flächendeckend Gruppenarbeit eingeführt, angesichts der hohen Arbeitsbelastung bleibt jedoch für Gruppengespräche oder selbstorganisierte Tätigkeiten der Arbeiter faktisch kein Raum, so daß sich jene die Gruppenarbeit ausmachende Spezifik letzten Endes auf die Zahlung einer Teamprämie reduziert. Der Vertreter des Betriebsrats schilderte in dem Interview sehr beredt den Widerspruch zwischen dem idealisierten Bild von der

10 „Es kann nicht das Ziel eines marktwirtschaftlich geführten Unternehmens sein, möglichst viele Mitarbeiter zu beschäftigten.“, meinte der Geschäftsführer des Werkes bereits 1994 lakonisch. 
Gruppenarbeit, das in den vorbereitenden Workshops gezeichnet wurde, und der praktischen Erfahrung. „Dann kommt die Realität des Lebens, weil sich halt nicht das im tagtäglichen Leben niederschlägt, was in Einführungsworkshops so wunderschön dargestellt wird. " In beiden Untersuchungsbetrieben dominieren traditionelle und aufgrund des knappen Personalbesatzes vor allem in dem ostdeutschen Untersuchungsbetrieb unter hohem Zeitdruck zu absolvierende Maschinenbedienung und Montagearbeiten mit geringen Möglichkeiten der Selbstorganisation. Die hierarchische Unterordnung des Shopfloors unter die disponierenden und kontrollierenden Funktionalbereiche bleibt erhalten, wenn auch in dem Betrieb OD/KFZ-1 gemäß dem „Small-Factory-Konzept“ eine prozeßnahe Integration von direkten und indirekten Funktionen stattfand und die traditionellen zentralen Stabsabteilungen Instandhaltung und Qualitätssicherung personell stark ausgedünnt und ihrer Weisungskompetenzen beraubt wurden.

\section{b) autonome Restrukturierung durch das betriebliche Management}

Im Unterschied zu den beiden oben beschriebenen Fällen OD/KFZ-1 und PL/KFZ-2 spielt die Lean-Programmatik in den anderen Untersuchungsbetrieben der Branche, in denen polnische, tschechische und ostdeutsche Manager weitgehend ohne Gestaltungsdirektiven der Eigentümer über die Unternehmens- und Arbeitsorganisation ihrer Betriebe befinden können, nur eine periphere Rolle. Fragen der Organisationsgestaltung erlangen in der Situationsdefinition der Manager überhaupt erst im Rahmen einer der folgenden Konstellationen den Stellenwert eines bewußt artikulierten Problems:

- Eine veränderte institutionelle Einbettung des Unternehmens macht organisatorische Veränderungen unmittelbar notwendig, um dessen Handlungsfähigkeit zu gewährleisten (z.B. der Aufbau einer betrieblichen Verwaltung in Betrieben, die früher im Rahmen größerer Wirtschaftseinheiten eine reine Fertigungsfunktion ausübten)

- Neue Technologien, Erzeugnisse oder größerer Personalabbau verlangen organisatorische Anpassungen, deren Unterlassung zu starken Störungen in der Funktionsweise der Organisation führen würde.

- Es treten aktuelle oder antizipierte Probleme auf, den Anforderungen des Marktes gerecht zu werden, die von den Managern mit der Gestaltung der Organisation in Verbindung gebracht werden. Die in Betracht gezogenen organisatorischen Veränderungen müssen einen klaren Beitrag zur Lösung dieser Probleme versprechen.

Da der Untersuchungsbetrieb OD/KFZ-2 früher eine reine Fertigungsstätte war, mußte zu Beginn des Transformationsprozesses zunächst eine eigene betriebliche Verwaltung einschließlich einer Konstruktionsabteilung aufgebaut 
werden, deren Kompetenzen hinsichtlich Kundenakquisition und Preisverhandlungen allerdings eingeschränkt sind, da diese Funktionen im wesentlichen durch die Mutterfirma wahrgenommen werden. Der Hersteller einfacher mechanischer Baugruppen ist einem harten Kostenwettbewerb ausgesetzt. Er reagierte darauf u.a. mit einem drastischen Personalabbau, der auch nichtintendierte Folgen für das Funktionieren der Unternehmens- und Arbeitsorganisation hatte. Der nunmehr kleinbetriebliche Charakter des Untersuchungsbetriebes erlaubt eine effiziente direkte Kontrolle betrieblicher Abläufe durch den fachlich kompetenten Geschäftsführer. Der knappe Personalbesatz bewirkte ein hohes $\mathrm{Ma} \beta$ an Aufgabenintegration bei den verbliebenen Führungskräften („Bei uns finden Sie nur Multifunktionäre“, OD/KFZ-2, Geschäftsführer), das eine bürokratische Abgrenzung verhindert und in Verbindung mit der langjährigen Betriebszugehörigkeit eine effiziente Kommunikation ermöglicht ${ }^{11}$ Die Krisenerfahrungen und die nach wie vor hohe Arbeitslosigkeit bewirkten eine hohe Bereitschaft der Mitarbeiter, ihr Verhalten im Interesse des Unternehmens zu optimieren, so daß die Entwicklung von Motivations- und Disziplinierungsmethoden keine Aufgabe war, der sich das Management stellen mußte. Die Kernbereiche der Arbeitsorganisation blieben im wesentlichen unverändert, allerdings erhielten die Arbeiter eine größere Eigenverantwortung bei der Qualitätssicherung und es erfolgten pragmatische Veränderungen der Arbeitsorganisation, die einer besseren Anpassung an die eingeführte neue Technik dienten. Gruppenarbeit wird nicht praktiziert, da die Manager wie auch der Betriebsrat von deren Nutzen für das eigene Unternehmen nicht überzeugt sind.

Die geschäftsführenden Eigentümer der beiden tschechischen Untersuchungsbetriebe kennen und schätzen nach eigenem Bekunden die modernen westlichen Konzepte organisatorischer Modernisierung. Der Eigentümer von TR/KFZ-1 ist ein tschechischer Emigrant, der auch aus eigener Erfahrung die Praxis der Branche im westlichen Ausland kennt. Er wendet dieses Wissen in seinem Unternehmen punktuell an, so wurde die Zahl der Leitungsebenen reduziert, einzelne Abteilungen wurden zusammengelegt, um Schnittstellen abzubauen, es handelt sich also vorwiegend um Veränderungen im Bereich der Verwaltung. In den beiden tschechischen Untersuchungsbetrieben blieben die produktionsbezogenen Kernbereiche der Arbeitsorganisation weitgehend unverändert, Gruppenarbeit wird nicht praktiziert, es gibt keine Bildung von Cost- oder Profitcentern. Trotz punktueller Anwendungen von Konzepten aus dem Bereich der Lean-Philosophie wurde im Untersuchungsbetrieb TR/KFZ-1, im

${ }^{11}$ Es wäre deshalb falsch, diese Art der Arbeitsorganisation einfach als „tayloristisch“ zu charakterisieren, da in diesem Fall die für die „wissenschaftliche Betriebsführung“ charakteristische strikte bürokratische Abgrenzung zwischen einzelnen betrieblichen Funktionalbreichen nicht gegeben ist. 
Unterschied zum Untersuchungsbetrieb OD/KFZ-1, deren Rationalisierungspotential kaum genutzt, die Belegschaft verringerte sich nur geringfügig auf der Basis natürlicher Abgänge. Der Geschäftsführer von TR/KFZ-1 hebt die große Bedeutung guter Beziehungen zur Belegschaft als Voraussetzung für die Realisierung seiner langfristigen unternehmerischen Vision hervor und versucht, die Mitarbeiter durch Prämienlöhne und durch ständige Information über betriebliche Angelegenheiten $\mathrm{zu}$ motivieren. Der Geschäftsführer des Untersuchungsbetriebes TR/KFZ-2 betont die große Bedeutung von weiter existierenden betrieblichen Sozialeinrichtungen und verbesserter Arbeitsbedingungen für die soziale Integration der Belegschaft und führte eine „Tafel des Stolzes“ ein, auf der vorbildliche Arbeitsleistungen öffentlich gewürdigt werden. Während die ostdeutschen und zum Teil auch die polnischen Manager in ihrer Situationsdefinition von einer weitgehend gegebenen sozialen Integration der Belegschaften in die Unternehmen und einem hohen Maß an Zumutbarkeit gegenüber der Belegschaft ausgehen, scheinen die tschechischen Manager eher die Notwendigkeit zu sehen, diese Integration durch ein behutsames Taktieren, durch die Betonung von Sozialpolitik und Unternehmenskultur erst zu erreichen. Die Gründe dafür sehen sie vor allem in der Arbeitsmarktsituation, die insbesondere Fachkräften aufgrund alternativer Beschäftigungsmöglichkeiten eine recht starke Position verleiht. ${ }^{12}$

Ungeachtet aller Veränderungen im Detail und trotz aller Unterschiede zwischen unseren Untersuchungsbetrieben fällt doch auf, daß die aktuellen Anforderungen seitens des Marktes hinsichtlich Flexibilität, Zeit, Kosten und Qualität durchaus auf der Grundlage einer Verbindung von moderner Technik und einer eher traditionell organisierten Produktion zu bewältigen sind. Der durchaus ernst gemeinte Versuch einer flächendeckenden Einführung von Gruppenarbeit im Untersuchungsbetrieb OD/KFZ-1 endete angesichts der Zeit- und Kostenzwänge damit, daß weitgehend unveränderte Verhältnisse auf der Shop-Floor-Ebene als „Gruppenarbeit" etikettiert wurden. ${ }^{13}$ In den Fällen, wo das betriebliche Management autonom handeln konnte, gab es deshalb für sie keine Veranlassung, die aus der Vergangenheit bekannten Organisationsformen der Massenproduktion grundsätzlich in Frage zu stellen. Der eher kleinbetriebliche

${ }^{12}$ Im Fall von TR/KFZ-1 wird dies noch durch die regionale Nähe des Unternehmens zur deutschen Grenze verstärkt, da der geschäftsführende Eigentümer auch mit Blick auf den EU-Beitritt eine Abwanderung von Fachkräften nach Deutschland befürchtet.

${ }^{13}$ Unsere Untersuchungsergebnisse stimmen durchaus mit Einschätzungen überein, daß unter den Bedingungen verstärkter Konkurrenz in der Automobilindustrie ein Trend zur ReZentralisierung und zur stärkeren Betonung standardisierter Arbeitsabläufe festzustellen ist (Schumann 1998, Springer 1998). Allerdings kann man in den ostdeutschen, tschechischen und polnischen Untersuchungsbetrieben weniger von einer Rücknahme sprechen, da es auf Selbstorganisation und Dezentralisierung setzende Arbeitsformen kaum gegeben hat. 
Charakter der Untersuchungsbetriebe und die in der Regel langjährige persönliche Bekanntschaft der Führungskräfte ermöglichte auch ohne institutionalisierte Formen der Funktionsintegration das notwendige Maß eines flexiblen und raschen Zusammenwirkens der betrieblichen Bereiche. Diese Kontinuität bedeutet nun nicht die Abwesenheit jeglicher Veränderung, von denen wir insbesondere zwei hervorheben möchten: In den Fällen einer leitbildorientierten organisatorischen Modernisierung durch die Mutterfirmen entstanden institutionalisierte Formen zur Nutzung des Produzentenwissens und zur Einbeziehung der Beschäftigten in den einzelnen Arbeitsplatz übergreifende Zusammenhänge in Form von Problemlösungsgruppen, Qualitätszirkeln u.ä. Weitere Veränderungen ergaben sich daraus, daß die Manager seitens der Kunden in durchaus „coerciver" Weise aufgefordert wurden, ihre Qualitätsmanagementsysteme zertifizieren zu lassen. Dies führte zur Etablierung eines dichteren Kontrollnetzes und zur Dezentralisierung von Qualitätsverantwortung. Arbeiter müssen nun vielfach vorgeschriebene Kontrollprozeduren selbst ausführen und die Korrektheit ihrer Arbeit durch ihre Unterschrift bestätigen.

\subsection{Maschinenbau}

Alle untersuchten Betriebe des Maschinenbaus haben in den 90er Jahren in (erzwungener) Abkehr von der Serienfertigung und der Belieferung von osteuropäischen Volumenmärkten eine Hinwendung $\mathrm{zu}$ überwiegend kundenorientierter bzw. kundenspezifischer Produktion vollzogen. ${ }^{14}$ Dies bedeutet eine dramatische Veränderung der externen Bedingungen und daraus resultierender Anforderungen hinsichtlich Flexibilität, Qualität und Kosten im Vergleich zur Planwirtschaft, deren Realisierung ohne größere organisatorische Veränderungen kaum gelingen konnte. Die Problematisierung überkommener organisatorischer Strukturen und Praktiken in der Situationsdefinition der Manager geht deshalb hier weiter als in den beiden anderen in die Untersuchung einbezogenen Branchen.

Die weitreichendsten Veränderungen in den Organisationsstrukturen der Maschinenbaufirmen fanden wir in Ostdeutschland. Bereits zu Beginn der 90er Jahre hatte es, zunächst noch unter Treuhandverwaltung, unter dem Zwang zur Kostenreduzierung angesichts ausbleibender Aufträge eine drastische „Verschlankung“ mit dem Abbau von Hierarchieebenen, der Zusammenlegung von Bereichen, Ausgliederungen peripherer Bereiche und Massenentlassungen gegeben. In der zweiten Häfte der neunziger Jahre ließen sich notwendige

${ }^{14}$ Mit Ausnahme des polnischen Betriebes PL/MB-2 ist für die Maschinenbaufirmen eine hohe Innovationsrate bei den Produkten typisch. Sie wird von den Managern als eine wichtige Bedingung für das Bestehen im Wettbewerb gesehen. 
Kosten- und Zeiteinsparungen ${ }^{15}$ in den ostdeutschen Betrieben des Maschinenbaus nicht mehr durch technische Modernisierung und weitere personelle Ausdünnung erzielen. Das Management begann nunmehr verstärkt nach organisatorischen Innovationen zu suchen. Im Ergebnis dessen schufen die Führungskräfte der ostdeutschen Betriebe (bei OD/MB-1 initiiert vom westdeutschen Eigentümer) Organisationsstrukturen, die insbesondere auf die Integration von Markt- und Kostenkriterien bereits in den Prozeß der Konstruktion und damit auch auf die Überwindung eines unter den Ingenieuren wie Mitarbeitern der Fertigung noch nicht gänzlich überwundenen Hanges zum technischen Perfektionismus abzielten, der dem Trend im Maschinenbau hin zu kostenbewußter Zweckmäßigkeit entgegenstand. ${ }^{16}$

Ungeachtet aller Unterschiede im Detail sind die entstandenen Organisationsstrukturen der untersuchten ostdeutschen Maschinenbaubetriebe auf eine größere Geschlossenheit der funktional verbundenen Prozesse, die Beseitigung von Schnittstellen, die Verkürzung von Entwicklungswegen, auf Kostensenkung und eine optimalere Arbeit mit den Kunden gerichtet.

Bei D/MB-2 erfolgte dies beispielsweise in Form zweier Produktgruppen. ${ }^{17}$ Integriert in jede Produktgruppe, die Cost-Centern vergleichbar und auf ein prozeßorientiertes Re-Engineering gerichtet sind, wurden die nur für das jeweilige Produkt zuständigen Bereiche Entwicklung, Konstruktion, Arbeitsvorbereitung, Montage und Verkauf. Die Fertigung bedient im Sinne eines internen Dienstleisters beide Produktgruppen. Die Produktgruppen werden von jeweils einem Produktgruppenmanager geleitet, dem die

${ }^{15}$ Wie empirische Untersuchungen ergaben, befinden sich ostdeutsche Unternehmen in einer Zwangssituation: Um sich auf neuen Märkten zu etablieren, müssen sie ihre Erzeugnisse in starkem Maße an die jeweiligen Kundenwünsche anpassen. Andererseits haben sie kaum die Möglichkeit, den dadurch bedingten Aufwand durch eine entsprechende Preisgestaltung zu kompensieren, was zu einem starken Kostendruck führt. (Ragnitz 1998)

${ }^{16}$ Die Vernachlässigung von Kostenkriterien und ein Hang zum technischen Perfektionismus galten bislang im deutschen Maschinenbau insgesamt als Schwachpunkte (vgl. Kalkowski 1996).

${ }^{17}$ Die Einführung der neuen Organisationsstruktur wurde verbunden mit der Inbetriebnahme des neuerbauten Firmengebäudes. Diese Investitionsmaßnahme hatte die Mutterfirma als notwendig angesehen, um ihre bis dahin an zwei Standorten operierenden ostdeutschen Tochterfirmen unter einem Dach vereinigen zu können. Nach Aussage des zur Familie des Eigentümers gehörenden westdeutschen Geschäftsführers war die Konzeption des neuen Fabrikgebäudes daran ausgerichtet, der neuen Struktur die räumlichen Voraussetzungen zu schaffen: „...ein Grundsatz, den wir hier verwirklicht haben, Transparenz, Großzügigkeit, Licht, Kommunikation. Also ich nenn's auch unbewußte Kommunikation, es ist ja hier Arbeitsvorbereitung, Konstruktion direkt im Bereich der Montage auf gleicher Ebene, nur durch Fenster getrennt, und Geschäftsführung auch im im gleichen Bereich, also sehr flache Hierarchien und sehr kurze Wege. 
Gesamtverantwortung obliegt, „daß wir also von der Angebotstätigkeit über den Verkauf bis hin zur Auslieferung der Maschine diesen gesamten Zyklus letztendlich in einer Hand konzentriert haben"(Produktgruppenmanager). Die Funktion des Meisters gibt es formal nicht mehr, allerdings nehmen die Vorarbeiter in der Fertigung und den Montagebereichen real einige traditionelle Meisteraufgaben mit wahr. ${ }^{18}$

Charakteristisch für die ostdeutschen Maschinenbaubetriebe ist vor allem die auf der Ebene des oberen Managements vollzogene Aufgaben- und Funktionsintegration, insbesondere zwischen Konstruktion und Vertrieb, die komplexe Erweiterung von Verantwortung und Zuständigkeiten der Führungskräfte sowie der über diesen Weg vollzogene Abbau von Schnittstellen zwischen Bereichen. Die Selbstorganisationsmöglichkeiten der Beschäftigten der ostdeutschen Betriebe in der Fertigung sind hingegen begrenzt geblieben mit allerdings deutlich erweiterter Eigenverantwortung für Fragen der Qualitätssicherung und erwarteter multifunktionaler Einsetzbarkeit -, die Aufgabenzuweisung erfolgt hierarchisch, wobei dies in hohem Maß den Erwartungen der Beschäftigten entspricht.

Im Unterschied zu der weitgehenden Funktionsintegration auf der Ebene des Managements in den ostdeutschen Firmen des Maschinenbaus, sind in den polnischen und tschechischen Untersuchungsbetrieben der Branche trotz Verkleinerung und Personalreduzierung die Organisationsstrukturen bislang stärker funktional ausdifferenziert und arbeitsteilig geblieben.

Die eher an großbetriebliche Strukturen erinnernde Arbeits- und Funktionsteilung in den tschechischen Maschinenbaubetrieben führt zwangsläufig zu bürokratischer Abgrenzung zwischen den Funktionsbereichen und mangelhafter Kommunikation ${ }^{19}$, was sich zunehmend als Hemmnis für eine flexible und kundenorientierte Produktion erweist. Die polnischen und tschechischen Manager lassen deshalb auch ein verstärktes Problembewußtsein hinsichtlich einer angemessenen organisatorischen Veränderung erkennen, das sich aber noch nicht in dem Maße wie in den ostdeutschen

${ }^{18}$ Bei OD/MB-2 wurden die Abteilungen Konstruktion und Vertrieb zu einem Bereich unter der Leitung eines Managers mit Prokura zusammengeführt, der zweite Bereich Produktion/Logistik umfaßt Arbeitsvorbereitung, Fertigung, Montage Einkauf und Logistik und wird ebenfalls von einem Manager mit Prokura geleitet. Direkt dem Geschäftsführer unterstellt ist die Qualitätssicherung.

${ }^{19}$ In den ostdeutschen Maschinenbaufirmen verweisen die Führungskräfte hingegen auf die problemlos und effizient gehandhabte Kommunikation und Kooperation zwischen den Funktionalbereichen, die eine nicht zu unterschätzende Ressource für die Gewährleistung der für kundenspezifische Produktion unabdingbaren Organisationsflexibilität darstellen und zweifellos zur positiven Entwicklung der Firmen beitragen. 
Untersuchungsbetrieben in Form von Lösungen niedergeschlagen hat. Die Manager des MbO-privatisierten Betriebes TR/MB-2 beabsichtigten beispielsweise zum Zeitpunkt unserer Erhebungen eine Reform in Richtung stärkerer Funktionsintegration und Prozeßmanagement. Angestrebt wird, eine Matrixstruktur „quer“ $\mathrm{zu}$ den weiter bestehenden traditionellen Leitungsstrukturen in Form von Projektteams zu bilden, die für die Realisierung konkreter Aufträge zuständig sind. Ob die hierbei vorgesehene doppelte Unterstellung (Teamleitung und Geschäftsleitung als „zweistufige Geschäftsleitung"') zu einer Vereinfachung der Abstimmungsprozesse und der innerbetrieblichen Kooperation führen wird, bleibt allerdings abzuwarten. Die in diesem Betrieb eine Zeit anhaltenden Qualitätsprobleme, die sich in einer entsprechend hohen Zahl von Reklamationen widerspiegelten, führten zu einer strukturellen Innovation, die allerdings unter den tschechischen Maschinenbaufirmen derzeit wohl eine Ausnahme bildet. Die Montage erfolgt heute unter Einbeziehung der Produktionsarbeiter direkt beim Kunden (d.h. es gibt keine interne Montageabteilung mehr), wodurch Zeit und Kosten gespart und die Reklamationen deutlich gesenkt werden konnten.

Auch in dem kommerzialisierten polnischen Großbetrieb PL/MB-1 tratenTendenzen bürokratischer Abschottung betrieblicher Funktionalbereiche auf, die noch durch organisatorische Probleme zwischen den seit 1995 formell eigenständigen Holdingbetrieben verstärkt wurden. Versuche des Managements, diese Probleme zu überwinden, scheiterten am Widerstand informeller Gruppen und Führer. Während die innerbetrieblichen Akteursbeziehungen in den ostdeutschen und tschechischen Betrieben überwiegend konfliktfrei und an Konsens orientiert sind und den Entscheidungen der Führungskräfte seitens der anderen betrieblichen Akteure in der Regel mit passiver Akzeptanz begegnet wird, sehen sich Manager in kommerzialisierten Großbetrieben in Polen bei betrieblichen Reformen oft dem aktiven Widerstand von Gewerkschaften und informellen Gruppen gegenüber. ${ }^{20}$

Das Management von PL/MB-1 löste dieses Problem mit der Einführung von Gruppenarbeit in den produzierenden Bereichen. Die Top-Manager und Abteilungsleiter als die Initiatoren dieser Reform begannen im Ergebnis einer strukturellen Analyse des Arbeitsprozesses 1996 (nach einer Versuchsphase 1995) Gruppenarbeit in allen Produktionsabteilungen des Betriebes einzuführen. Diese arbeitsorganisatorische Veränderung wurde verbunden mit einer Neugestaltung des Entlohnungssystems, das mit einem Bewertungssystem zur Einschätzung der Arbeit der Beschäftigten verknüpft wurde. Die Teams sind

${ }^{20}$ In Betrieben mit einem privaten Eigentümer ist der Einfluß der Gewerkschaften, so es ein Gewerkschaftskomitee überhaupt gibt, unbedeutend. Entscheidungen des Managements stoßen hier in der Regel nicht auf Widerstand. 
autonome Kollektive, die ca. 15 Personen umfassen und selbständig, ohne Einflußnahme durch die Leitung, einen Teamführer wählen. Auch die Auswahl der Team-Mitglieder können sie selbst vornehmen. In die Kompetenz des Teamführers fällt die Zuweisung von Aufgaben, die Kontrolle der Ausführung der Tätigkeiten sowie die Zuteilung der Prämie, die von anfangs $10 \%$ auf $30 \%$ erhöht wurde. Die Gruppenarbeit erweiterte nach Einschätzung der Manager real die Handlungsspielräume der Mitarbeiter.

Die Manager, die Gruppenarbeit im Ausland kennengelernt hatten, begründeten deren Einführung zwar unter Berufung auf das Human-Ressource-Konzept, den Ausschlag gab indes zweifellos die anders vom Management kaum konfliktfrei $\mathrm{zu}$ bewältigende, so aber mögliche Integration der informellen Führer. Die letztlich als Resultat mikropolitischen Taktierens eingeführte Gruppenarbeit schuf einen Zwang zur Kooperation und paralysierte den starken Widerstand der Gewerkschaften gegen eine Differenzierung der Löhne, da die Mitarbeiter die Aufteilung der Prämie nun selbst regeln.

Wenngleich die polnische Firma PL/MB-2 mit nur 124 Beschäftigten sehr viel überschaubarer und hinsichtlich des Grades der funktionalen Ausdifferenzierung eher den ostdeutschen Betrieben vergleichbar ist, zeigten sich auch in diesem Betrieb Probleme in der Zusammenarbeit. Angesichts des drohenden Bankrotts der Firma verbanden die Manager die Einführung der Gruppenarbeit gleichsam mit der Hoffnung auf Rettung durch Selbstorganisation der Beschäftigten, als einen letzten Versuch zur Mobilisierung aller Reserven. Die Gruppenarbeit hat, u.a. mit der Wahl des Gruppenführers, partizipative Elemente. Alle internen Akteure bewerten die Gruppenarbeit sehr positiv, insbesondere die in dieser Firma sehr gut qualifizierten Arbeiter gelten als deren „glühende Verfechter“, da die Gruppenarbeit ihnen die Möglichkeit bietet, ihre Qualifikation optimal zu nutzen, auch wenn man mit dem Entlohnungssystem keinen zusätzlichen Anreiz zu Gruppenarbeit gesetzt sieht.

Die Möglichkeit bzw. Sinnhaftigkeit von Gruppenarbeit wird von den Managern in den ostdeutschen Maschinenbaubetrieben dagegen eher skeptisch beurteilt. „....machen wir nicht, Gruppenarbeit. Ich sag, wir sind 'ne große Gruppe. Wir machen auch Gruppenarbeit, nur mit einer mit 100 Mitarbeitern (...) Man darf nicht überorganisieren." - so der Geschäftsführer von OD/MB-2. Der westdeutsche Geschäftsführer von OD/MB-1 könnte sich Gruppenarbeit in der Fertigung später durchaus vorstellen, sieht aber in der gering ausgeprägten Bereitschaft der Beschäftigten zur Übernahme von mehr Verantwortung ein Hindernis, dessen Abbau erst noch eines Lernprozesses bedürfe. Auch in den untersuchten tschechischen Firmen war Gruppenarbeit kein Thema. Die Existenz von Gruppenarbeit allein vermag allerdings in den polnischen Maschinenbaubetrieben - ungeachtet ihrer positiven Effekte - nichts an der schlechten wirtschaftlichen Situation der Firmen zu ändern, wenn, wie in den 
untersuchten Fällen, Investitionsmittel zur Erneuerung der veralteten Technik fehlen, Auftragsmangel herrscht, Gewerkschaften unpopuläre, aber aus der Sicht der Manager notwendige Maßnahmen zur Kostenreduktion verhindern und der Handlungsspielraum der Firmen durch die Eigentümer restriktiv begrenzt wird. ${ }^{21}$

\subsection{Textil- und Bekleidungsindustrie}

Die Arbeits- und Organisationsstrukturen in den untersuchten Betrieben der Textil- und Bekleidungsbranche weisen in allen drei Untersuchungsländern eine große Übereinstimmung auf. Es überwiegt heute eine klein- und mittelständische Struktur in dieser Branche, was sich auch in der Auswahl unserer Projektbetriebe widerspiegelt. Alle Betriebe weisen eine traditionelle Organisationsstruktur auf. Mit drei Leitungsebenen (Geschäftsführer, Abteilungsleiter, Meister) sind sie relativ flach und damit überschaubar. Mit Ausnahme von TR/MB-2 sind die Eigentümer auch die Geschäftsführer, Entscheidungsprozesse sind damit in hohem Maße zentralisiert.

Die Arbeitsorganisation zeichnet sich überall durch eine weitgehende Spezialisierung aus: „Wir verdienen unser Geld damit (...) indem wir Leute spezialisieren auf ihre Maschinen und auf die damit möglichen Arbeitsgänge, so. Ich will gar net, daß die Leute ganze Teile nähen von hinten bis vorne, das ist unsinnig, vollkommen unsinnig. Ich kriege die Routine nur rein durch Übung des Gleichen “. (aus den alten Bundesländern stammender geschäftsführender Eigentümer von OD/TB-1) Die Aufgabenzuweisung erfolgt strikt hierarchisch. Eine Dezentralisierung und Aufgabenerweiterung hat es in der Qualitätssicherung gegeben: die Qualitätskontrolle liegt sowohl in der Verantwortung der Beschäftigten selbst als auch bei den Abteilungsleitern. Unabhängig davon bestehen überall Verantwortliche oder Abteilungen für die Qualitätssicherung.

In allen Betrieben der Branche beurteilten die Manager länderübergreifend die bestehenden Arbeits- und Organisationsstrukturen als optimal und alternativlos hinsichtlich ihrer Effektivität, um den Anforderungen des Marktes gerecht zu werden. Qualität, Zuverlässigkeit und das schnelle, flexible Eingehen auf die Wünsche der Kunden, verbunden mit einem entsprechenden Service - darin sehen die Manager der Textil- und Bekleidungsbranche in den drei Untersuchungsländern mit hoher Übereinstimmung das Erfolgsrezept ihres

21 Formal privatisierten polnischen Staatsbetrieben (Eigentümer sind hier staatliche Institutionen oder Betriebe) wie z.B. den in unsere Untersuchung einbezogenen Maschinenbaufirmen billigte man bislang keine Perspektive $\mathrm{zu}$, diese Eigentumsform wurde als ein Übergangsstadium zur „eigentlichen“ Privatisierung gesehen. Inzwischen deutet sich hier ein Umdenken an. 
guten Bestehens am Markt. Die strategische Bedeutung, die in den Betrieben der Branche der Marktarbeit zugemessen wird, zeigt sich u.a. darin, daß in allen Firmen (mit Ausnahme von TR/TB-2) diese Aufgabe von den geschäftsführenden Eigentümern selbst wahrgenommen wird. Alternativen zu den bestehenden Arbeits- und Organisationsstrukturen, wie sie die neuen Managementkonzepte anbieten, sind aus Sicht der Eigentümer und Manager hinsichtlich Zeit, Kosten, Flexibilität und Produktivität den traditionellen Strukturen unterlegen und finden daher keine Anwendung. ${ }^{22}$ Generell verzeichneten wir eine ausgeprägte Skepsis gegenüber den in der Managementliteratur offerierten westlichen Organisationskonzepten. So meinte der geschäftsführende Eigentümer von PL/TB-1, diese seien nützlich für Großbetriebe, in kleinen Firmen wie seiner reichten Fähigkeiten des Rechnens, Konsequenz und Intuition, verbunden mit dem gesunden Menschenverstand.

Der überall in den Nähbereichen zugrundegelegte Akkordlohn wird von den Managern in dieser Branche als das effektivste und gerechteste Instrument für die dauerhafte Gewährleistung einer hohen Arbeitsmotivation und -disziplin gesehen. „... also ich sag', wenn jeder sein eigner ist, und jeder sehen muß, daß nachmittags auf der Lohnkarte was drauf steht, dann ist das besser, als wenn ich jetzt sag': Ihr seid 'ne Gruppe und ihr macht mal jetzt das. " (Produktionsleiterin $\mathrm{OD} / \mathrm{TB}-2)$

Um den Kundenanforderungen bezüglich Qualität ${ }^{23}$ und Flexibilität entsprechen zu können, nutzt man im Rahmen der bestehenden Strukturen folgende Ressourcen:

- ständige Optimierung aller Phasen der Auftragsrealisierung, u.a. durch den Einsatz moderner Rechentechnik in der Produktionsplanung, Informationsgewinnung und Logistik

- unbürokratisch gehandhabte bereichsübergreifende Kommunikation unter den Führungskräften in den kleinen Betrieben

${ }^{22}$ In beiden polnischen Bekleidungsfirmen gibt es in einzelnen Produktionsabteilungen technologisch bedingte Arbeitsgruppen, die allerdings, obwohl als Gruppenarbeit bezeichnet, keine partizipativen und auf Selbstorganisation sowie Kompetenzerweiterung gerichteten Elemente enthalten und nicht über das traditionelle Muster der Organisation hinausreichen.

${ }^{23}$ Die Schwierigkeiten, infolge der wirtschaftlichen Krise zu Beginn der 90er Jahre die für die hohen Qualitätsansprüche der westlichen Kunden hochwertigen Stoffe zu beschaffen, bewogen den polnischen Bekleidungsbetrieb PL/TB-1 dazu, fortan nur noch Bekleidung aus den von den Kunden zur Verfügung gestellten Materialien zu produzieren. 
- höchste Anforderungen an die Gewährleistung der Qualität über ein System der Kontrolle und Selbstkontrolle; einige Betriebe sind zertifiziert ${ }^{24}$

- Kooperation mit anderen Firmen im In- und Ausland bzw. Gründung von Tochtergesellschaften

- Zugriff auf die in allen untersuchten Betrieben der Branche gegebene Bereitschaft der Beschäftigten zu Überstunden, um flexibel auf die Anforderungen des Marktes und diskontinuierliche Auftragsauslastung zu reagieren. ${ }^{25}$

- Beibehaltung einer hohen Arbeitsintensität in allen Betrieben, wachsende Auftragsvolumen werden mit im wesentlichen gleichbleibenden Belegschaftsstärken realisiert. ${ }^{26}$

- große Akzeptanz- und fehlende Konfliktbereitschaft der - in dieser Branche zumeist weiblichen - Belegschaften; vor allem in Ostdeutschland und Polen wird angesichts der schwierigen Arbeitsmarktsituation dem Erhalt der Arbeitsplätze erstrangige Bedeutung vor eventuellen anderen Forderungen beigemessen.

\section{Fazit}

Fragen der Gestaltung von Arbeits- und Organisationsstrukturen waren in allen untersuchten Transformationsländern Teil eines sehr komplexen betrieblichen Reformgeschehens, denen in den meisten Fällen keine herausgehobene Bedeutung gegenüber anderen, im Verständnis der Manager und Eigentümer zunächst eher dringlicheren Themen wie etwa Markterschließung, Produktinnovation, technische Modernisierung, Zugang zu Kapital u.a.

${ }^{24}$ Der hohe Anteil an Lohnveredlung bzw. Lohnfertigung für westeuropäische Abnehmer in der polnischen und tschechischen Bekleidungsindustrie, der in beiden Ländern wegen der damit zwangsläufig verbundenen Abhängigkeit von den Auftraggebern auch kritisch bewertet wird, unterstellt die Firmen den harten Qualitätsanforderungen und -parametern der Abnehmer

${ }^{25}$ Tschechische und polnische Arbeiter sehen darin eine willkommene und z.T notwendige Aufstockung ihres Lohnes, in den ostdeutschen Betrieben werden Überstunden überwiegend in Zeiten geringerer Auftragsauslastung, die oft saisonal bedingt sind, abgesetzt, es gibt Arbeitszeitkonten.

${ }^{26}$ In den polnischen Betrieben erhöht das Management in solchen Fällen die Normen. Daß inzwischen eine Grenze der Zumutbarkeit erreicht ist, wird von den Managern der ostdeutschen Betriebe und den Führungskräften auf unterer und mittlerer Ebene in den polnischen Betrieben vermerkt, Neueinstellungen in größerem Umfang lehnen die Geschäftsführer aus Kostengründen und z.T. wegen schwankender Auftragslage aber ab. 
zugemessen wurde. Sie gewannen dann an Aufmerksamkeit, wenn die gegebenen Strukturen und Funktionsweisen der Organisation der Bewältigung extern gesetzter Anforderungen entgegenstanden. Das aber heißt auch: Wo die überkommenen Arbeits- und Organisationsstrukturen sich als angemessen erwiesen, die aus einer marktwirtschaftlich geprägten Umwelt entstehenden Anforderungen erfolgreich zu bewältigen, gab es für das Management keine Veranlassung zu Veränderungen, die über die unvermeidlichen Anpassungen an die neuen Rahmenbedingungen hinausgingen. Erfolgreich heißt dabei für die Führungskräfte, das dauerhafte Bestehen des Betriebes am Markt zu sichern und ein positives betriebswirtschaftliches Ergebnis zu erzielen. Das durch die Erfahrung gestützte Vertrauen zu der Funktionalität eines überkommenen Grundbestandes organisatorischer Lösungen auch unter veränderten Bedingungen war für die Manager ein Stabilitätsmoment in einer sich vielfach dramatisch verändernden Umwelt mit neuartigen Problemen. Geradezu symptomatisch hierfür stehen in den untersuchten Ländern die Betriebe der Textil- und Bekleidungsindustrie. Individueller Akkord bei weitgehender Spezialisierung von Arbeitsaufgaben und Mehrmaschinenbedienung in der Weberei und Spinnerei gelten den Eigentümern und Managern in dieser Branche nach wie vor als alternativlose Leitbilder einer effizienten Organisation, mit denen sich, wie ihre Erfahrungen zeigen, auch die neuen, ungleich höheren Flexibilitäts- und Qualitätsanforderungen gut bewältigen lassen. Ähnlich gilt dies für die auf Massenproduktion ausgerichteten Betriebe der Kraftfahrzeugzulieferindustrie, wo ebenfalls traditionelle Formen der Arbeitsorganisation überwiegen.

Im Maschinenbau hingegen hatten die Unternehmen auf dem Weg zu einer organisationalen Handlungsfähigkeit einen wesentlich höhere Reformbedarf zu bewältigen, da die großbetrieblich organisierte Produktionsweise von Maschinen für die Volumenmärkte des RGW, wie sie unter den Bedingungen der Planwirtschaft üblich war, durch eine auf individuelle Kundenwünsche ausgerichtete Qualitätsproduktion abgelöst werden mußte. Der dadurch bedingte drastische Anstieg der Flexibilitäts- und Qualitätsanforderungen seitens des Marktes veranlaßte die Manager in allen Untersuchungsbetrieben der Branche zur Suche nach Organisationslösungen, die jene für die Realisierung kundenspezifischer Aufträge unverzichtbare Flexibilität der Zusammenarbeit der Bereiche und Elastizität des Produktionsprozesses gewährleisten sollten.

Wenngleich jeder Untersuchungsbetrieb eine individuelle Konstellation von strukturellen Handlungsbedingungen, durch die Manager vorgenommenen Situationsdefinitionen und manageriellem Handeln zur Erlangung der organisatorischen Handlungsfähigkeit im Transformationsprozeß aufweist, zeichnen sich, wie unsere Untersuchung zeigte, dennoch typische Muster innerhalb der einbezogenen Branchen $a b$, die allerdings durch länderspezifische 
Besonderheiten modifiziert werden. Diese Länderspezifik, die die organisationsrelevante Definition von Problemen durch die Manager in gewisser Weise vorprägt, resultiert im wesentlichen aus den unterschiedlichen Transformationspfaden, die ab 1990 auf der Makroebene eingeschlagen wurden (vgl. Hanke/Mense-Petermann 1997; speziell zu den Eigentumsumwandlungen: Brinkmann et al. 1999). Es kann an dieser Stelle nur auf einige Spezifika eingegangen werden.

- Die für Ostdeutschland 1990 gewählte ordnungspolitische Option, die zu einer unvermittelten Aussetzung der Betriebe an Marktbedingungen unter Beibehaltung der Hochlohnoption führte, unterwarf die Firmen branchenübergreifend um den Preis ihrer Existenz einem radikalen Druck auf Kostensenkung. Einem solch massiven Anpassungsdruck, der "schlanke“ Betriebe mit flachen Hierarchien und minimalem Personalbesatz nachgerade erzwang, waren weder die tschechischen noch die polnischen Betriebe ausgesetzt, und bis heute sind die im Vergleich zu Ostdeutschland niedrigen Löhne in Polen und Tschechien ein offensichtlicher Vorteil für das Bestehen am Markt. Damit war für die Manager dieser Länder freilich auch nicht gleichermaßen der Zwang zu (kostenminimierenden) organisatorischen Veränderungen gegeben.

- Die eingeschlagenen Transformationspfade hatten unterschiedliche Auswirkungen auf die nationalen Arbeitsmärkte. Die in Ostdeutschland unverändert zwischen $18 \%-20 \%$ liegende offizielle Arbeitslosenrate, Ergebnis der infolge der „Schocktherapie“ einsetzenden Deindustrialisierung, muß als Hintergrund der hohen Anpassungs-und Leistungsbereitschaft aller Beschäftigten bei einer ständig steigenden Arbeitsintensität gesehen werden. Das Management kann, wie die Betriebe der Textil/Bekleidungs- sowie der Kraftfahrzeugzulieferindustrie zeigen, bislang problemlos den markt- und branchenbedingten Flexibilitätsanforderungen unter Zugriff auf dieses Potential gerecht werden, ohne daß es grundlegender Reorganisationen in den Arbeitsstrukturen bedarf. Das gilt ähnlich auch in Polen, wenngleich die Arbeitslosigkeit mit ca. $11 \%$ (bei allerdings deutlichen regionalen Unterschieden) hier geringer ist. Dem gegenüber bietet der tschechische Arbeitsmarkt bei einer noch immer relativ niedrigen Arbeitslosenrate den Beschäftigten, die zudem nicht jene akuten Krisenerfahrungen wie beispielsweise ostdeutsche Arbeitnehmer machen mußten, Alternativen, wenn sie sich arbeitsbedingten Zumutungen entziehen wollen. Angesichts eines verbreiteten Fachkräftemangels, der insbesondere im Maschinenbau akut ist (Vlacil u.a. 1996), gehen tschechische Manager organisationale Veränderungen sehr vorsichtig an oder verzichten ganz auf sie, wenn sie (passiven) Widerstand der Beschäftigten vermuten. 
- Einfluß auf die organisationsrelevante Problemdefinition der Manager haben auch die im Verlauf der Transformation entstandenen Muster der betrieblichen industriellen Beziehungen. Während in Ostdeutschland Betriebsräte und Belegschaften betrieblichen Reorganisationsmaßnahmen des Managements mit passiver Akzeptanz begegnen, zwingt die in polnischen Betrieben in Staatsbesitz in der Regel starke Position und Konfliktbereitschaft der Gewerkschaften die Manager, Reorganisationsmaßnahmen zu vermeiden, die zu Nachteilen für die Beschäftigten insbesondere der gewerblichen Bereiche führen können. Das eröffnet möglicherweise stärker als in Betrieben mit anderen Privatisierungsformen die Chance zu partizipativ angereicherten Reorganisationen der Arbeitsstrukturen, wie die Einführung von Gruppenarbeit im Ergebnis mikropolitischen Taktierens der Manager in einem polnischen Großbetrieb des Maschinenbaus zeigt, was allerdings allein nicht die wirtschaftlichen Probleme dieses Unternehmens zu lösen vermag. In Tschechien führt die traditionell fehlende Konfliktbereitschaft der Manager (Vlacil 1995) eher $\mathrm{zu}$ einem Hinauszögern und Vermeiden „schmerzhafter“ Reorganisationsmaßnahmen.

Mit Ausnahme der zwei in Konzernbesitz befindlichen Kraftfahrzeugzulieferfirmen, in denen es durch die Eigentümer zu einer leitbildorientierten Reorganisation kam, gingen die Führungskräfte in den Betrieben unseres Samples pragmatisch-problemorientiert, ausgehend von den branchenspezifischen Anforderungen des Marktes, an Fragen der Organisationsgestaltung heran. Die Einstellungen der von uns befragten Manager $\mathrm{zu}$ modernen westlichen Leitbildern der Organisationsgestaltung reichen dabei von dezidierter Ablehnung über Gleichgültigkeit bis zu der pragmatischen Integration einzelner Elemente, von denen sich die Manager einen konkreten Nutzen für die Lösung betrieblicher Probleme erhoffen, wie etwa im Maschinenbau. Mit Ausnahme der international agierenden Konzerne und einer westdeutschen Mutterfirma im Maschinenbau überließen die Eigentümer, so sie nicht ohnehin gleichzeitig Geschäftsführer wie in den meisten Textil- und Bekleidungsfirmen waren, den Managern die Entscheidung über die Gestaltung der Organisations- und Arbeitsstrukturen. Etwas zugespitzt könnte man die These formulieren, daß pragmatisch-problemorientiertes Handeln der Manager im Transformationsproze $\beta$ in der Textil-/Bekleidungsindustrie und in der Kraftfahrzeugzulieferindustrie zu einer Reproduktion eher traditioneller Organisationsformen der Massenproduktion führte, während das gleiche Handeln im Maschinenbau aufgrund der extern gesetzten hohen Flexibilitätsanforderungen die Entstehung durchaus innovativer Formen der Funktionsintegration zur Folge hatte.

Das pragmatische Handeln der Manager war den konkreten Bedingungen des Transformationsprozesses angemessen, bot es doch die Chance eines schnellen 
Reagierens auf vielfach drängende Probleme, zudem stand die allgemeine Unsicherheit über künftige Entwicklungen einem konzeptionellen Nachdenken über die längerfristige Erschließung organisatorischer Ressourcen entgegen. Angesichts neuer Herausforderungen an die Unternehmen in den Transformationsländern, wie sie sich beispielsweise aus der verstärkten Einbeziehung in die internationale Arbeitsteilung ergeben, könnte eine strategische Sicht der Manager auf die Probleme der betrieblichen Organisation einen neuen Stellenwert erhalten.

\section{Literatur}

Esser, H. (1996): Die Definition der Situation, in: Kölner Zeitschrift für Soziologie und Sozialpsychologie, Heft 1; S. 1-34.

Hanke, J./Mense-Petermann, U. (1997): Wirtschaftsreformen und betriebliche Restrukturierung. Polnische und tschechische Transformationspfade im Vergleich. Projektbericht, Erlangen- Nürnberg

Kalkowski, P. (1996): Innovationsstrategien des deutschen Maschinenbaus - Traditionelle Stärken, neue Herausforderungen, Ansätze zur Bewältigung, in: SOFI-Mitteilungen, Nr. 24, S.71-82.

Lungwitz, R.-E. (1997): Marktinduzierte und eigentümerinduzierte Restrukturierung unterschiedliche Muster der Transformation in Betrieben des ostdeutschen Maschinenbaus und der Automobilindustrie, in: Dittrich, E./Fürstenberg, F./Schmidt, G. (Hrsg.): Kontinuität im Wandel. Betriebe und Gesellschaften Zentraleuropas in der Transformation, München und Mering, S.61-70.

Lungwitz, R.-E/Preusche, E. (1999): Vom Mängelwesen zum Macher? Manager in Ostdeutschland als Gestalter einer leistungsfähigen Unternehmens- und Arbeitsorganisation, erscheint in: Arbeit, Heft 4

Mickler, O., Engelhardt, N., Lungwitz, R.-E., Walker, B. (1996): Nach der Trabi-Ära: Arbeiten in schlanken Fabriken, Berlin

Ragnitz, J. (1998): Determinanten der Produktivitätslücke in Ostdeutschland, in: Wirtschaft im Wandel, Heft 1, S. 3-11.

Schienstock, G. (1993): Management als sozialer Prozeß, in: Ganter, H.-D., Schienstock, G. (Hrsg.): Management aus soziologischer Sicht, Wiesbaden

Schumann, M. (1998): Frißt die Shareholder Value-Ökonomie die Modernisierung der Arbeit?; in: Hirsch-Kreinsen, H/Wolf, H. (Hrsg.): Arbeit, Gesellschaft, Kritik. Orientierungen wider den Zeitgeist, Berlin

Springer, R. (1998): Das Ende neuer Produktionskonzepte? Rationalisierung und Arbeitspolitik in der deutschen Automobilindustrie; in: Hirsch-Kreinsen, H./Wolf, H. (Hrsg.): Arbeit, Gesellschaft, Kritik. Orientierungen wider den Zeitgeist. Berlin

Tänzler, D. (1998): Radikaler Bruch und lange Dauer: Zur Rückkehr der Geschichte in Osteuropa am Fall der Tschechischen Republik, in: BISS public, Heft 25, S. 7-32. 
Vlacil, J. (1995): Arbeitskonflikte und industrielle Beziehungen in den privatisierten tschechischen Betrieben, in: Bieszcz-Kaiser, A./Lungwitz, R.-E./Preusche, E. (Hrsg.): Industrielle Beziehungen in Ost und West unter Veränderungsdruck, München und Mering, S. 107-121.

Vlacil, J./Hradecka, I./Mazalkova, I./McDermott, G. (1996): Politics, Skills and Industrial Restructuring, Working Papers 8, Institute of Sociology, Praha

Windolf, P./Brinkmann, U./Kulke, D. (1999): Warum blüht der Osten nicht? Zur Transformation der ostdeutschen Betriebe, Forschungen aus der Hans-BöcklerStiftung, Bd. 13 